\title{
Significados del bachillerato en jóvenes de colonias periurbanas marginadas en Yucatán, México
}

Meanings of the high school in young people from marginalized periurban neighborhoods in Yucatan, Mexico

\author{
Volumen 20, Número 1 \\ Enero - Abril \\ pp. 1-25
}

\section{Norma Graciella Heredia Soberanis}

\section{Citar este documento según modelo APA}

Heredia Soberanis, Norma Graciella. (2020). Significados del bachillerato en jóvenes de colonias periurbanas marginadas en Yucatán México. Revista Actualidades Investigativas en Educación, 20(1), 1-25. Doi. 10.15517/aie.v20i1.40132 


\title{
Significados del bachillerato en jóvenes de colonias periurbanas marginadas en Yucatán, México
}

Meanings of the high school in young people from marginalized peri-urban neighborhoods in Yucatan, Mexico

\section{Norma Graciella Heredia Soberanis ${ }^{1}$}

\begin{abstract}
Resumen: El presente artículo reporta los hallazgos de una investigación realizada con alumnado proveniente de un contexto en desventaja socioeconómica y cultural de colonias marginadas en Yucatán México. Debido a la relevancia social que tiene estudiar un bachillerato para conseguir un empleo o para continuar estudios de nivel superior, el objetivo de la investigación consistió en explorar los diversos significados y sentidos con respecto a estudiar, permanecer y concluir la educación media superior, esto con base en la experiencia escolar de jóvenes de un Bachillerato General Comunitario. El tipo de investigación es un estudio de caso con enfoque cualitativo, como informantes clave participaron 27 estudiantes: 23 en dos grupos focales y 4 en entrevistas individuales; la metodología utilizada para analizar los datos consistió en el análisis sociológico de los discursos. Como resultados relevantes se encontró que el bachillerato adquiere un significado de instrumento para lograr a futuro la superación personal y el progreso económico e intelectual; en referencia a la dimensión distributiva de la justicia social: tener un buen empleo y un buen sueldo son factores percibidos como necesarios para lograr el reconocimiento social; a su vez, la educación es vista como un instrumento válido y legítimo para su obtención, constituyendo ideológicamente un instrumento de lucha social de jóvenes en desventaja socioeconómica y cultural, para el logro de un modelo hegemónico de vida adulta; sin embargo, esto implica un individualismo enmascarado de liberación solidaria. De igual manera, se identificó la representación de nociones de injusticia social y educativa que normalizan la redistribución desigual de bienes y servicios; la falta del reconocimiento social hacia grupos vulnerados; y el énfasis en el consumo neoliberal.
\end{abstract}

Palabras clave: justicia social, justicia educativa, política educativa, evaluación curricular

\begin{abstract}
This article reports the findings of an research carried out with students from a context of socioeconomic and cultural disadvantage of marginalized colonies in Yucatan Mexico, given the social relevance of studying a baccalaureate in order to obtain employment or continue higher education; the objective was to explore various meanings and senses with regard to studying, remaining in and completing higher secondary education, based on the school experience of young people from a General Community High School. The type of research is a case study with a qualitative focus; 27 students participated as key informants: 23 in two focus groups and 4 in individual interviews; the methodology for analyzing the data consisted of sociological analysis of the speeches. As a result it was found that the high school acquires the meaning of an instrument to achieve personal improvement and economic and intellectual progress in the future. Referring to the distributive dimension of social justice: having a good job and a good salary are factors perceived as necessary to achieve social recognition, so that education is seen as a valid and legitimate instrument to obtain them, ideologically constituting an instrument of social struggle of young people in socioeconomic and cultural disadvantage, for the achievement of a hegemonic model of adult life, presenting a masked individualism of solidarity liberation. There is evidence of the representation of notions of social and educational justice that normalize the unequal redistribution of goods and services, the lack of social recognition of vulnerable groups, and the emphasis on neoliberal consumption.
\end{abstract}

Key words: social justice, educational justice, educational policies, curriculum evaluation

\footnotetext{
1 Profesora de la Universidad Autónoma de Yucatán, México. ORCID https://orcid.org/0000-0003-2995-0408
}

Dirección electrónica: nheredia@correo.uady.mx

Artículo recibido: 26 de junio, 2019

Enviado a corrección: 2 de octubre, 2019

Aprobado: 18 de noviembre, 2019 


\section{Introducción}

\subsection{Antecedentes}

En México, durante los últimos dos sexenios presidenciales del siglo XXI, las mejoras sustanciales a la calidad y a la pertinencia educativa, así como el combate al rezago educativo, han sido parte de la política nacional. La Secretaría de Educación Pública (SEP), estableció en el año 2007, en el Programa Sectorial de Educación 2007-2012: "Ampliar las oportunidades educativas para reducir desigualdades entre grupos sociales, cerrar brechas e impulsar la equidad" (p. 11); para esto, la SEP propuso acciones tales como la ampliación de la cobertura, así como el apoyo al ingreso y a la permanencia del estudiantado en la escuela. Para el caso de la Educación Media Superior, desde el año 2008 se inició la integración de un Sistema Nacional de Bachillerato (SNB), mediante la homologación de un perfil de egreso que permite a los estudiantes una Certificación Nacional de Educación Media Superior.

A finales de ese mismo año, una universidad pública del Estado de Yucatán (UDS) sumó sus esfuerzos educativos para formar parte de ese sistema, mediante la capacitación del profesorado en el enfoque basado en competencias. En esta misma línea, el 31 de agosto del 2009, dicha universidad creó el Bachillerato General Comunitario (BGC), el cual surgió, según Echazarreta (2009), para satisfacer las necesidades de ampliación de la oferta educativa, debido al aumento de población, así como para atender a jóvenes en estado de vulnerabilidad económica, provenientes de escuelas con poco acceso a una educación de calidad y tecnologías (Heredia y Peniche, 2016).

La escuela que ofrece el BGC está ubicada en una colonia localizada en la periferia del sur de la ciudad de Mérida, Yucatán, México (Universidad Autónoma de Yucatán, 2010). Como parte de su implementación, el proceso de selección de estudiantes de nuevo ingreso es diferente al realizado por las otras dos escuelas preparatorias de la UDS, ya que cuenta con una etapa de preselección que toma en cuenta las respuestas de sus aspirantes a un Cuestionario Socioeconómico y a una Prueba de motivación para el estudio; posteriormente, los aspirantes preseleccionados avanzan a una siguiente etapa que consiste en sustentar el EXANI I, examen que igualmente presentan los aspirantes de las otras escuelas preparatorias. El plan de estudios del BGC también fue diseñado de forma diferente al de las otras escuelas de nivel medio superior del Estado, ya que además de poseer un componente propedéutico, en este plan de estudios se integran además los componentes de inserción comunitaria y de capacitación para el trabajo (Heredia y Peniche, 2016). 
Con base en las características mencionadas del BGC, la presente investigación tuvo como objetivo: Explorar los diversos significados y sentidos con respecto a estudiar, permanecer y concluir un bachillerato universitario, en relación a la justicia social y educativa en un marco político de responsabilidad social, desde la experiencia de jóvenes que cursan un programa escolar en un contexto social de desventaja económica y cultural. En cuanto a la justificación de esta investigación, se comprende que el BGC fue creado para jóvenes con características sociales homogéneas, tales como pertenecer a un grupo socioeconómico catalogado como "en desventaja" social y de "bajo nivel cultural"; este programa educativo, representa un dilema: pues si bien se presenta un elemento de justicia, al reconocer la existencia de jóvenes aspirantes a cursar un bachillerato que no poseen condiciones sociales y académicas similares a los jóvenes de los niveles socioeconómicos medios 0 altos, razón por la que se establece un proceso de selección diferente al del bachillerato general de otras escuelas preparatorias de la misma universidad; la creación de una escuela con un currículo diferenciado, evidencia la segregación social.

En el contexto educativo, la diversidad necesita ser analizada y reflexionada en su dimensión ética como en el entendimiento de sus fines; tanto en el contenido del currículo, la pedagogía empleada, la organización y la estructura de las escuelas, así como en las prácticas de evaluación, resulta indispensable aclarar el significado de lo debe ser entendido como diversidad en la educación (Gimeno, 2002), por este motivo es sumamente relevante analizar y comprender el efecto de las políticas y de las acciones que conlleva realizar una nueva propuesta educativa con un currículo diferenciado.

A un año de su inauguración, durante el primer y segundo semestres, el departamento de servicios estudiantiles de la UDS encontró problemas como ausentismo y deserción escolar de los estudiantes del BGC. De 196 estudiantes que ingresaron en la primera generación, sólo 35 (17.85 \%) lograron graduarse en el año 2012, y aunque a partir del 2013 hasta el 2018, estas cifras han mostrado leves incrementos, sería necesario analizar longitudinalmente el funcionamiento de este bachillerato en cuanto a sus procesos educativos así como los fenómenos sociales que inciden en estas problemáticas de ausentismo y deserción, haciendo una valoración de si los egresados de enseñanza secundaria que viven en comunidades de áreas vulneradas de la capital del Estado, necesitan un currículo diferenciado con características de interacción comunitaria para el logro de la justicia social en sus vidas. 


\section{Referente teórico}

Entre las teorías para analizar la justicia social se encuentra la de Marx (1972) con su amplia crítica al capitalismo como un sistema que conduce a la enajenación del ser humano. En cuanto a la justicia educativa, la pedagogía crítica de Paulo Freire (2002) ha marcado una pauta histórica en su argumentación sobre cómo la deshumanización es el resultado de la opresión. Por su parte, las aportaciones de sociólogos críticos como Bourdieu y Passeron (1998) han enfocado el estudio de la escuela como uno de los principales medios reproductores de la violencia simbólica. Estas teorías analizan el impacto de las estructuras sociales sobre la ideología, y por tanto, en los diversos ámbitos y contextos de la vida de los seres humanos, entre ellos el educativo.

En 1995, Rawls analiza instituciones sociales en cuanto a la forma en que distribuyen los derechos y deberes fundamentales, así como la división de las ventajas de la cooperación social. Este último término se vincula directamente con el concepto de justicia, siendo indispensable establecer primero el ideal de cooperación social, en cuanto a sus fines y propósitos. La concepción general de justicia de Rawls (1995) enfatiza que: "todos los valores sociales $\square$ libertad y oportunidad, ingreso y riqueza, bases del respeto a sí mismo $\square$ habrán de ser distribuidos igualitariamente, a menos que una distribución desigual de alguno o de todos estos valores redunde en una ventaja para todos" (p. 69).

Otro pensamiento alternativo para pensar en la justicia, basado en el desarrollo humano, consiste en el enfoque de capacidades propuesto por Sen (1995), entendiendo éstas como un conjunto de vectores de funcionamientos, que reflejan la libertad de cada persona para llevar un tipo de vida u otro (p. 20); este enfoque ha sido empleado a manera de marco normativo-conceptual para valorar el bienestar social, la calidad de vida y las políticas para lograr ambos. En esta propuesta, las capacidades son un aspecto clave para que cada persona pueda ejercer su libertad para seleccionar la forma de vida que prefiera, siendo justo el hecho de considerar las oportunidades que se hayan tenido para ser o tener ciertas propiedades o cosas; por lo tanto, la pobreza, con base en esta perspectiva, consiste en la privación de capacidades básicas y no sólo como la falta de ingresos (Sen, 2000, p. 114).

Basándose en la reflexión filosófica, epistemológica y política, Fascioli (2011) menciona que las dimensiones ética y moral de la justicia social, aportan una visión ampliada de dicho concepto, como guía de las prácticas sociales, adicionando a los problemas de distribución, 
el de la falta de reconocimiento, por lo que la identidad y la indiferencia son el trasfondo de prácticas sociales injustas.

El concepto de justicia, ha evolucionado desde la noción de distribución y redistribución de bienes y servicios, a un concepto más complejo en el que se implica el desarrollo de las capacidades humanas y el reconocimiento social de la dignidad de cada persona, como base para el análisis y la generación de políticas sobre derechos y obligaciones para la convivencia, teniendo como condición la libertad individual y la solidez de instituciones del Estado, como las escuelas, para ejercer plenamente dichos derechos, tomando en cuenta impedimentos o problemas que pudieran presentar personas pertenecientes a grupos vulnerados.

En cuanto al contexto educativo, como parte del ejercicio de la justicia, Connell (2006) menciona que se pueden encontrar problemas en cuanto a la honestidad de la distribución, apuntando al concepto de igualdad, ya que es difícil, por ejemplo, imaginar cómo un grupo social puede merecer más o menos educación que otro, puesto que no es una simple cuestión de derechos individuales (justicia procedente de Platón), sino que el equilibrio y la armonía deben presidir la vida social que es compartida; por tanto, la igualdad individual es la condición de un orden social justo, no su objetivo (p. 25).

Según Bolívar (2012, pp. 14-17) la justicia, entendida como igualdad y aplicada al aspecto educativo, se refiere a una diversidad de interpretaciones prácticas, de las que se identifican cuatro tipos de nociones:

1. Igualdad de oportunidades (o de acceso): En el sentido político, consiste en nivelar a las personas para que se encuentren en las mismas condiciones, a través de acciones y recursos compensadores. Implica compensar todas las circunstancias de las que la persona no es responsable, como por ejemplo, la condición familiar. Asimismo, existe la propuesta de no compensar aquello de lo que la persona sí es responsable y forma parte de su esfuerzo personal, voluntad y libre elección. A su vez, existen críticas acerca de que dicho esfuerzo no es independiente de los condicionamientos sociales, lo que significa que la nivelación es imposible.

2. Igualdad de enseñanza: En esta noción, el centro escolar establece la diferencia en la calidad de la enseñanza y del aprendizaje, buscando garantizar una buena educación y buenos aprendizajes, sin discriminar a las personas. La igualdad de enseñanza consiste en otorgar una enseñanza de buena calidad al alumnado, para ello se requiere de una oferta de contenidos curriculares, experiencias de aprendizaje y estrategias de 
enseñanza, valiosas culturalmente y en un marco de política educativa que potencie la igualdad. Sin embargo, el logro de la justicia bajo esta idea, requiere que todas las escuelas tengan idénticas condiciones e idéntica calidad de enseñanza, lo cual es imposible.

3. Igualdad de conocimiento y éxito escolar: Tiene por finalidad evitar la reproducción de desigualdades, siendo necesaria la introducción del principio de la diferencia, enfocándose en el alumnado con mayor desventaja, para así contrarrestar los efectos negativos del modelo meritocrático de igualdad de oportunidades. Este principio de discriminación positiva consiste en una justicia distributiva que tenga en cuenta desigualdades reales para compensar a todo lo que escapa a la responsabilidad individual.

4. Igualdad de resultados: Parte de la idea de que el alumnado, independientemente de su origen social, tiene la probabilidad de aprender las mismas cosas en un nivel determinado. Asimismo, la igualdad de consecuencias educativas, se refiere a que los alumnos con similares resultados educativos tienen las mismas oportunidades sociales de acceso al mercado de trabajo y a otras posiciones sociales, y por tanto a tener vidas similares como resultado de su escolarización; sin embargo, resulta necesario reconocer que el sistema escolar no es autónomo, debido a la articulación entre la escuela y el destino social.

En relación a lo expuesto anteriormente, propuestas de autores críticos como Gimeno (2002), Martínez (2005), Connell (2006), Torres (2009) y Bolívar (2012), entre otros, hacen evidente que las decisiones políticas en relación con los derechos de cada persona, afectan la educación recibida como derecho social, de acuerdo a las diferentes etapas de vida y los diversos contextos sociales.

En investigaciones recientes realizadas en escuelas, autores como Hernández-Castilla, Euán e Hidalgo (2013) han analizado la promoción de la justicia social por parte del personal directivo, encontrando dentro de las características relevantes de un líder, el compromiso por una educación justa, la capacidad de reflexión, así como la apertura y la accesibilidad para comunicarse con todos los agentes del centro escolar. En las escuelas, el aprendizaje del alumnado no es ni periférico ni opcional, sino imprescindible. El conocimiento enseñado no es neutral ni objetivo, sino que está determinado por los intereses humanos y refleja las relaciones sociales, económicas y políticas de la sociedad. Las escuelas son instituciones 
sociales que plantean cuestiones morales, éticas, sociales, filosóficas e ideológicas, que no pueden ser tratadas como si estuvieran basadas en valores neutrales y libres de ideología (Cochran-Smith y Demers, 2009, pp. 274 -275). Por lo tanto, es relevante investigar en relación a las nociones de la educación como derecho para el logro de justicia social, las ideologías que subyacen en el alumnado proveniente de contextos vulnerados. Sobretodo en el contexto del presente siglo XXI, en el que los efectos tanto positivos como negativos que ha traído la globalización bajo la ideología neoliberalista, nos llevan a retomar a nivel internacional y local, la necesidad de hacer efectiva la justicia social en todos los países del mundo.

\section{Metodología}

\subsection{Enfoque}

Esta investigación es un estudio de caso interpretativo, que muestra los resultados finales del trabajo de campo realizado en diciembre del año 2014. Con base en Simons (2011) y Stake (2007), se optó por realizar un estudio de caso bajo el enfoque cualitativo, dado el uso de un diseño de investigación emergente, flexible y abierto.

\subsection{Población de estudio}

El estudio de caso fue desarrollado en una escuela pública de nivel medio superior localizada en el sur de la ciudad de Mérida, región metropolitana y capital del Estado de Yucatán, México. El sur de la ciudad de Mérida cuenta con habitantes cuyas viviendas están construidas con madera, láminas de cartón o plásticos, y quienes carecen de servicios básicos tanto dentro como fuera de sus casas; igualmente presentan transporte urbano insuficiente y poco acceso a créditos bancarios para la construcción de viviendas.

El alumnado de la escuela es de nivel socioeconómico bajo; sus padres trabajan de manera informal como albañiles o vendedores ambulantes y las madres como empleadas domésticas. Para la presente investigación, la selección del alumnado participante fue intencional, por conveniencia, siendo un total de 23.

Como parte de la primera etapa en el campo, fueron realizados dos grupos focales con los 23 estudiantes de dos cohortes: 1. Grupo focal realizado con 13 estudiantes (7 mujeres y 6 hombres) de la primera generación del bachillerato que habían finalizado el tercer semestre del bachillerato y que se encontraban asistiendo al curso remedial de la asignatura de Desarrollo del lenguaje algebraico; 2. Grupo focal efectuado con 10 estudiantes (4 mujeres y 
6 hombres) de la segunda generación del bachillerato, quienes habían finalizado el primer semestre y asistían al curso remedial de la asignatura: Desarrollo del Pensamiento Intelectual.

En una segunda etapa del estudio, fue realizada una entrevista individual con estudiantes que obtuvieron el promedio de calificación más alto de la primera generación del bachillerato. Finalmente, como parte de esta misma etapa de recolección de datos, fueron realizadas dos entrevistas individuales con dos estudiantes (1 mujer y 1 hombre) en situación de estudiantes irregulares, y en riesgo de ser dados de baja del bachillerato por adeudar asignaturas.

En la tabla 1 se muestra la relación de estudiantes participantes en los grupos focales y en las entrevistas individuales mencionadas, con los cuales se hizo la validez de las interpretaciones, quienes a través de su consentimiento informado, autorizaron emplear sus diálogos para la ejemplificación de dichas interpretaciones.

Tabla 1. Participantes del proyecto en la etapa de validez de interpretaciones

\begin{tabular}{|c|c|c|c|c|}
\hline $\begin{array}{c}\text { Código de } \\
\text { participante }\end{array}$ & Edad & Sexo & Semestre/generación & Grupo \\
\hline \multicolumn{5}{|c|}{ Primera etapa de campo, grupo focal 1} \\
\hline R.O.P. & 19 & Hombre & Tercero/ primera & $\begin{array}{l}\text { Curso remedial Desarrollo } \\
\text { del lenguaje algebraico. }\end{array}$ \\
\hline R.M.P. & 19 & Mujer & Tercero/ primera & $\begin{array}{l}\text { Curso remedial Desarrollo } \\
\text { del lenguaje algebraico. }\end{array}$ \\
\hline J.V.C. & 20 & Hombre & Tercero/ primera & $\begin{array}{l}\text { Curso remedial Desarrollo } \\
\text { del lenguaje algebraico. }\end{array}$ \\
\hline M.T.A. & 20 & Mujer & Tercero/ primera & $\begin{array}{l}\text { Curso remedial Desarrollo } \\
\text { del lenguaje algebraico. }\end{array}$ \\
\hline \multicolumn{5}{|c|}{ Primera etapa de campo, grupo focal 2} \\
\hline R.E.F. & 19 & Hombre & Primero/ segunda & $\begin{array}{l}\text { Curso remedial Desarrollo } \\
\text { del } \quad \text { pensamiento } \\
\text { intelectual. }\end{array}$ \\
\hline L.R.M. & 18 & Mujer & Primero/ segunda & $\begin{array}{l}\text { Curso remedial Desarrollo } \\
\text { del } \\
\text { intelectual. }\end{array}$ \\
\hline M.A.C. & 18 & Hombre & Primero/ segunda & $\begin{array}{l}\text { Curso remedial Desarrollo } \\
\text { del } \\
\text { intelectual. }\end{array}$ \\
\hline I.P.C. & 18 & Mujer & Primero/ segunda & $\begin{array}{l}\text { Curso remedial Desarrollo } \\
\text { del } \\
\text { intelectual. }\end{array}$ \\
\hline \multicolumn{5}{|c|}{ Segunda etapa de campo, entrevistas individuales } \\
\hline M.T.R. & 19 & Hombre & Irregular/ segunda & Irregular \\
\hline M.A.F. & 20 & Hombre & Egresado/ primera & Mejor promedio \\
\hline C.M.R & 20 & Mujer & Egresada/ primera & Mejor promedio \\
\hline
\end{tabular}

Fuente: elaboración propia, 2019 


\subsection{Técnicas de recolección}

Las técnicas empleadas para la recogida de datos fueron: observación no participante, grupos focales, entrevistas individuales, análisis de documentos y diario de la investigadora. Los instrumentos que sirvieron para recolectar los datos consistieron en el llenado de un formulario de registro anecdótico para la observación no participante, un guión de preguntas estímulo para los grupos focales y un guión para las entrevistas individuales, cuyos cuestionamientos estuvieron dirigidos a comprender las razones, motivos, significados y sentidos que tuvo el bachillerato para cada estudiante, en tres momentos de su experiencia escolar: al ingresar al bachillerato, al estar cursándolo y al concluirlo. En el caso del análisis de documentos, se hizo la lectura del plan de estudios del bachillerato, así como la revisión de los programas de estudio de las asignaturas. Adicionalmente a estas técnicas e instrumentos, la comprensión del caso también implicó la asistencia a conferencias impartidas al alumnado y la observación del ambiente social en el centro escolar: interacciones entre el alumnado dentro y fuera del aula, formas de relacionarse en los pasillos, en la cafetería y en situaciones de juego.

\subsection{Procesamiento de análisis}

Para realizar el análisis cualitativo de los datos fue empleada la metodología de Conde (2010) denominada análisis sociológico de los discursos, debido a que aporta una propuesta innovadora que emplea como unidad de análisis el corpus de textos de la investigación en su conjunto, dicho análisis cualitativo fue realizado con apoyo del software Atlas.ti versión 8.4.

El procedimiento de análisis basado en Conde (2010) consistió en la lectura del corpus de textos, entendiendo texto como la transcripción de cada registro anecdótico, entrevista individual, grupo focal y diario de la investigadora; también fueron realizadas anotaciones en dichos textos a partir de la lectura de los mismos en el software Atlas.ti versión 8.4, así como la realización de procedimientos para interpretar y analizar el sistema de discursos, para la posterior redacción del informe de resultados (ver figura 1). 
Figura 1

Proceso general seguido en la investigación para el análisis sociológico del sistema de discursos

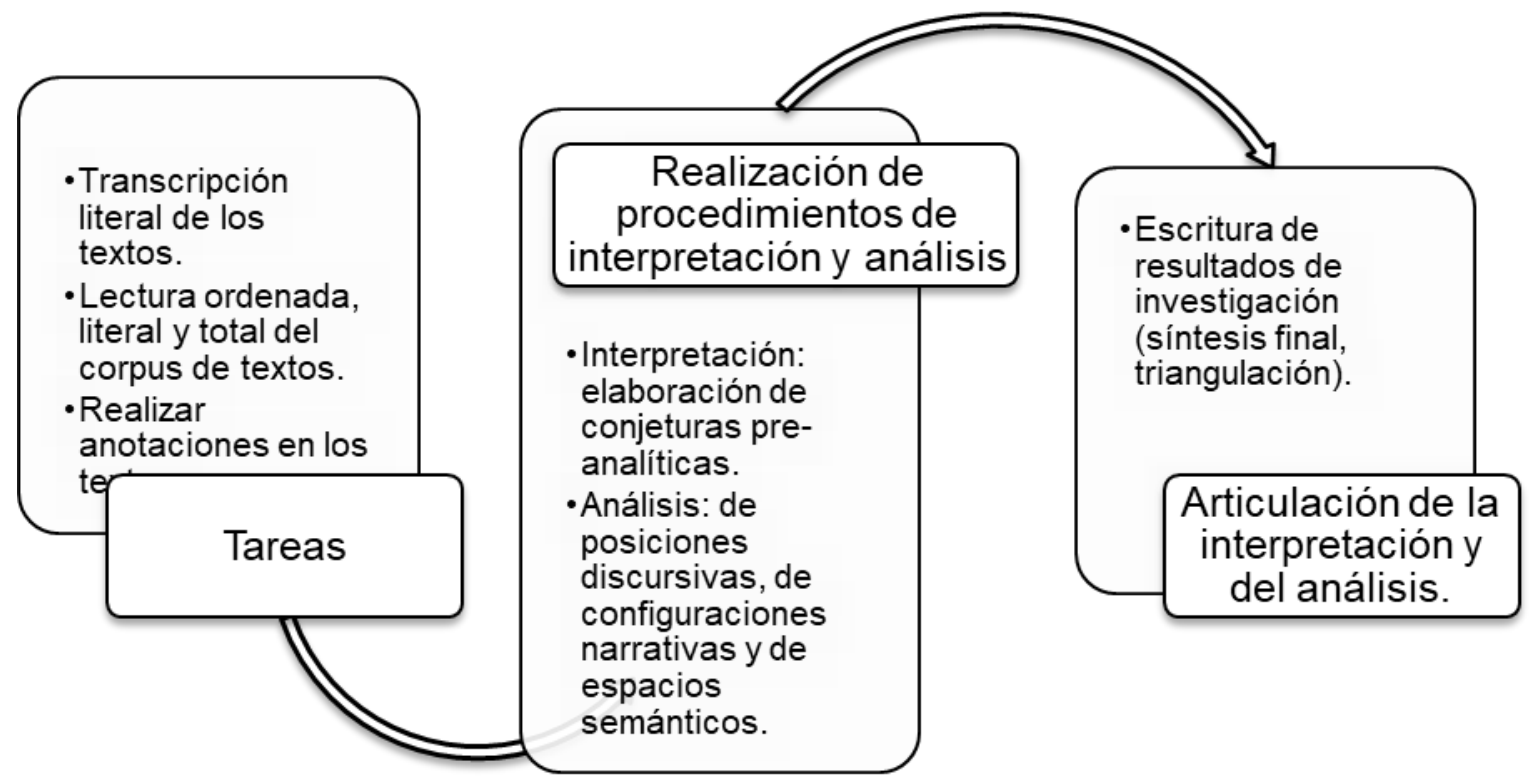

Fuente: Elaboración propia con base en la información del libro metodológico de Conde (2010).

\section{Resultados}

Con base en los significados, sentidos y motivaciones expresados por el alumnado participante acerca de estudiar, permanecer y concluir el bachillerato, se encontró que el tiempo futuro juega un papel fundamental, siendo una noción temporal caracterizada por la idea del progreso que se espera lograr en la vida adulta; tal como expresa R.O.P., quien comentó que estudia el bachillerato para: "Poder tener una carrera y poder sustentarme en algo en un futuro, si me quedaba sólo con la secundaria, qué iba a conseguir de empleo". Con la misma línea de pensamiento, M. A. C. mencionó que estudia para: "Tener una profesión, o sea, poder ser algo en la vida, o seguir siendo, para poder realizar nuestros sueños de ser profesionistas, abogado, un doctor o algo así por el estilo". Por su parte, M.T.R. también expresó que ingresó al bachillerato para estudiar una carrera universitaria, ser alguien y mejorar su nivel socioeconómico.

Con base en las declaraciones citadas anteriormente, se observa que el bachillerato adquiere un sentido utilitario al ser visto como un medio para tener una profesión; de la misma manera, la expresión "tener una profesión" se encuentra estrechamente ligada a la idea de "ser algo" o "ser alguien". A su vez, el significado otorgado a la idea de ser algo o 
alguien, consiste en ser una persona que ha logrado en su vida adulta la independencia respecto de los padres, y que a diferencia de éstos, ha concluido una carrera universitaria para desempeñar en la sociedad el rol de profesionista. Es decir, bajo esta forma de pensar, ser joven cobra importancia en la sociedad, en cuanto se haya superado tal estado perteneciendo a un grupo adulto de élite que posee una carrera universitaria, desempeña una profesión y es independiente de sus padres.

El "tener" para poder "ser" refleja, con base en Marx (1972), la forma en la que el modo de producción capitalista basado en el trabajo enajenado, aliena al hombre haciéndolo extraño de su propio cuerpo, dejando fuera de él su naturaleza, su ser espiritual, su esencia humana; es decir, el capitalismo convierte y determina a los seres humanos, sean trabajadores o capitalistas, en una mercancía humana; y los produce como seres deshumanizados física y espiritualmente (pp. 113-125). Al respecto, Freire (2002) menciona que la deshumanización no es un destino dado, sino el resultado de un orden injusto que genera violencia de los opresores sobre los oprimidos, quienes han sido despojados de su palabra, comprados en su trabajo, lo que significa la venta de la persona misma y en consecuencia, el ser menos (pp. 32-33). De esta manera, la violencia de los opresores queda manifestada por las personas en etapa adulta, pues debido a la estructura social y cultural, son quienes ostentan el poder sobre los menos poderosos, en este caso, las personas en la etapa de juventud. Desde este enfoque, en el contexto del sistema escolar, el poder es otorgado al personal directivo y al profesorado, ambos en etapa adulta, sobre los menos poderosos, es decir, el alumnado en etapa juvenil.

Según Tinoco y Evangelista (2006), la edad, o mejor dicho, las etapas de vida, son referentes de diferenciación que colocan a la juventud en una posición desigual dentro de la jerarquía social. Ser joven en una clase social, grupo étnico, o traslocalidad determinada, implica ser portador de varias identidades que remiten a una gama de oportunidades y limitantes. Se ha construido un modelo hegemónico desde el cual han sido definidos los mecanismos tradicionales de incorporación a la vida adulta: aun cuando en la juventud se es apto biológica y psicológicamente para ejercer el protagonismo social, éste es otorgado por la sociedad hasta que la persona sea considerada como un adulto racional, responsable y controlable; por lo tanto, mientras esto se logra, las personas en etapa de juventud, son sometidas a la tutela de las instancias adultas. A su vez, la expectativa social sobre la juventud, es que la principal actividad de cada joven sea la realización de los estudios, en condición de dependencia de sus padres o familiares, esta expectativa excluye de la 
definición de joven, a aquellas personas, sobretodo de contextos rurales, que dentro de esa misma etapa de juventud, ya han iniciado su vida laboral, conyugal y/o reproductiva.

Por otro lado, ante el estatus de subordinación y de indefinición, expuesto anteriormente, pocas veces cada joven es reconocido por el grupo adulto como otro [igual]; siendo mayormente considerados como personas sujetadas, con posibilidad de tomar algunas decisiones, pero no todas; con capacidad de consumir pero no de producir; con potencialidades para el futuro pero no para el presente (pp. 176-183).

Las categorías sociales relacionadas con el estatus que otorgan la participación productiva, estableciendo divisiones jerárquicas entre los jefes/empleados $u$ obreros/profesionistas, también han sido incorporadas en la ideología juvenil, ya que en los textos analizados fue manifestada la preferencia por pertenecer a identidades de categorías sociales dominantes. Como ejemplo de estas representaciones, se encuentra lo expresado por R. E. F.:

Pues en especial... igual quiero, quiero ser un profesionista, no quiero, pues... no quiero ser, por ejemplo, un, sin ofender, un obrero o algo así, porque va a ser difícil ofrecerle a mi familia lo que siempre he querido, lo que siempre le prometí a mi mamá.

Igualmente, C. M. R. comentó que estudió y concluyó el bachillerato porque:

Tengo ejemplos a mi alrededor, a mis vecinos, mi familia, amigos que dejan de estudiar y se dedican a trabajar y muchas veces como que pierden su vida por así decirlo, y yo no quería eso para mí. [Ella aclara a qué se refiere con perder la vida] Porque ... por ejemplo, en la secundaria tenía compañeras que ya tenían novio y pues como que era un objeto de distracción por así decirlo, porque yo veía que se iban a fiestas, se iban a pasear, se iban a otros lugares y dejaban de hacer tareas y dejaban de ser responsables, y yo me ponía a pensar, yo no quiero eso para mí, porque no voy a lograr ser nada importante si yo no tengo estudios, entonces eso me motivó a no dejar la academia y a no seguir ejemplos que para mí eran como que malos.

Como puede observarse, en los textos citados se encuentra la idea de que una persona sin estudios, en comparación con otra que sí los tiene, no posee el mismo valor. Esta forma de interpretar el valor humano: a mayor grado de estudios, mayor valor como persona, está vinculada a la idea de ser algo o alguien; en otras palabras, si se poseen 
estudios universitarios, entonces una persona cobra importancia en la sociedad, pues ha adquirido el valor necesario para ser y existir socialmente.

El considerarse menos y anhelar el ser más, en palabras de Freire (2002) consiste en guardar en sí mismos la conciencia opresora, pues ser equivale a parecerse al opresor, logrando ser 'el hombre nuevo', un hombre transformado en subopresor u opresor de otros, un hombre con una visión individualista que le imposibilita la conciencia de sí como persona y la conciencia como clase oprimida (pp. 35 - 39). De esta forma, se observa cómo en los textos analizados se está reproduciendo inconscientemente una ideología que lleva a la repetición del ciclo opresor - oprimido; ideología necesaria para mantener la estructura socioeconómica de dominación que genera injusticia; así se observa que ha sido incorporado el individualismo, fragmentándose de esta manera, la naturaleza social necesaria para la liberación del sí mismo en conjunto con el otro.

El modelo hegemónico de vida adulta incorporado, está basado en el logro del estatus de profesionista (ser médico, abogado, entre otras profesiones universitarias con prestigio social y sueldos bien remunerados). Por lo tanto, la búsqueda de ascenso social, entendido éste como el proceso por el cual siendo descendiente de una determinada clase social, se pasa a una clase superior a la de origen, es una idea que funciona como incentivo para concluir como meta intermedia, el bachillerato. Tener una carrera profesional es una forma de evitar en el futuro, pertenecer a una clase obrera o vivir en una situación de pobreza; por lo tanto, se puede decir que en la ideología reproducida por el alumnado, la educación continúa representando un medio que permite la movilidad social en ascenso: estudiar es el instrumento de lucha social utilizado por la juventud para la superación personal y el logro de una vida adulta exitosa. La educación proporcionada en el bachillerato, desde esta perspectiva, es un bien capitalizable, entendiendo como bien de capital, aquél cuya utilidad consiste en producir otros bienes, o que contribuyen directamente a la producción de los mismos.

La ideología capitalista se ve representada en el pensamiento de cada joven, a través de los motivos o razones que expresaron acerca de estudiar el bachillerato; precisamente esta reproducción de ideología, hace posible que los grupos hegemónicos continúen ejerciendo el control social para desarrollar la producción, por lo que bajo esta estructura socioeconómica se convertirán, como menciona Ornelas (2007), en el número creciente de trabajadores intelectuales asalariados que requieren los dueños de los medios de producción para la administración eficiente en todos los niveles de las instituciones, incluyendo las 
instituciones gubernamentales encargadas de los asuntos administrativos y económicos de los integrantes de la clase dominante y su fracción hegemónica (pp. 30-31).

Vinculado con lo comentado en el párrafo anterior, Bourdieu y Passeron (1998) ya habían advertido que:

Lejos de ser incompatible con la reproducción de la estructura de las relaciones de clase, la movilidad de los individuos puede concurrir a la conservación de estas relaciones, garantizando la estabilidad social mediante la selección controlada de un número limitado de individuos, por otra parte, modificados por y para la ascensión individual, y dando así su credibilidad a la ideología de la movilidad social que encuentra su forma más perfeccionada en la ideología escolar de la Escuela liberadora. (p. 225)

En este mismo sentido utilitarista, otro de los significados asignados al bachillerato, consiste en entenderlo como un medio para incrementar el capital cultural con el que se cuenta, esto coincide con el pensamiento alternativo de Sen (1995), que propone que cada estudiante ejerce su libertad para seleccionar su forma de vida considerando las oportunidades que tiene para el propio desarrollo humano; tal como comenta R. M. P.: el bachillerato "Me ha dejado muchos conocimientos, valores... conocimientos, pues con respecto a conocer más sobre las ciencias... sobre la historia, y cosas así... que enriquecen la cultura de uno".

El pensamiento citado también representa la ideología transmitida por la comunidad académica del centro escolar, lo que forma parte del currículo oculto del bachillerato, en donde el incremento del capital cultural constituye una de las metas universitarias; en concordancia a lo que señala Bourdieu (1997), la conformación del habitus cultural es una forma del capital consistente en la socialización sucesiva de las personas para su desenvolvimiento en público. De esta forma, la educación que ofrece el bachillerato también es entendida como un instrumento de poder para cada joven que ha tenido acceso a él, pues les empodera para abrirse puertas por sí mismos; es decir, es un bien otorgado para la tan anhelada igualdad como principio de la justicia (Rawls, 1995; Bolívar, 2012). Entonces, bajo esta ideología, la educación contribuye al progreso racional; según Popkewitz (2000), el colocar la esperanza en que la acción racional pueda provocar el progreso, lleva consigo supuestos epistemológicos que vinculan dicho progreso con la razón, la autocrítica y la intervención humana con las instituciones sociales. Recordemos que la llustración y la 
aparición del Estado nacional, tuvieron como efecto que las personas fueran provistas de una identidad colectiva, que es a la vez anónima y concreta:

Se hizo posible considerar a las personas de forma específica y detallada, como poblaciones categorizables en subgrupos, en cierta medida distintos de lo que constituye el todo. El concepto de población permitió la aparición de nuevas técnicas de control, dado una mayor posibilidad de supervisión, observación y administración del individuo; esta modernidad tiene la esperanza de crear situaciones sociales más democráticas, equitativas y justas relacionadas con la producción de la escolarización de masas y sus formas modernas de disciplina. Por medio de la escolarización de masas se civiliza a los individuos y se ejerce poder sobre ellos. El conocimiento del progreso se convirtió en una práctica social que vinculaba problemas de poder con la construcción de identidades. (Popkewitz, 2000, pp. 45-50)

De esta forma, el saber enseñado en las escuelas constituye una forma de ejercer el poder por parte de los grupos hegemónicos, quienes seleccionan la base conceptual que habrá de permear el pensamiento, desde la formación del profesorado hasta la educación del alumnado; un saber que posibilita el conocimiento y la identificación con identidades sociales.

Según Bernstein (1998) el conocimiento transmitido en la escuela está basado en un principio distributivo, de forma que los diversos conocimientos son repartidos de maneras distintas en los diferentes grupos sociales, y no están basados en diferencias neutras entre los saberes, sino en una distribución del conocimiento que transmite un valor, un poder y un potencial desiguales. De esta manera, la formación del alumnado no es sólo disciplinar, sino ideológica, y la ideología impregnada en los discursos refleja la expectativa social de identificación con los grupos dominantes de la estructura social.

Las ideologías representadas en los textos citados, reproducen la desvalorización de los rasgos distintivos de los sectores sociales en condición de pobreza, constituyendo una ideología cargada de injusticia social hacia la dignidad de las personas. De esta manera, se genera un anhelo o necesidad, según Honnet (como se citó en Cortés, 2005), por parte de las personas, de tener un efecto positivo en la sociedad, de estar incluido y de ser reconocido dentro de ésta mediante una lucha por el reconocimiento, dimensión moral de la justicia social (p. 10). El alumnado lucha por un reconocimiento social, del cual se creen carentes debido a su identificación con la etapa de la juventud, que institucionalmente es 
socializada como una clase social en desventaja. La lucha del alumnado por el reconocimiento consiste en la conclusión del bachillerato y en el logro del ingreso a una carrera universitaria; no obstante, en lo comunicado por los participantes, no se identifican comentarios que expresen el reconocimiento explícito de la dignidad de las personas que no cuentan con estudios universitarios, lo que implica que consideran a estas personas como “menos”, en relación a quienes sí cuentan con estudios universitarios.

Aunado a esto, la frase "mejorar la calidad de vida" estuvo presente en varios relatos de los jóvenes, quienes expresaron que el bachillerato constituye un medio para mejorar su calidad de vida actual. Con respecto a lo que significa una mejor calidad de vida, M. A. F. expresó: "Es tener más, o sea, contar ... con un sustento económico, o sea, ... ayudar a mi familia más que nada y pues igual mejorar mi calidad de vida en el sentido económico, tener más posibilidades económicas". Por su parte, R. M. P. aseveró que estudió el bachillerato para ser profesionista, porque:

Pues yo quiero mejorar mi calidad de vida, la de mi familia... quiero poder darle a mi familia en un futuro lo que tal vez ahorita no me pueden dar mis papás... a pesar de que a mí no me hace falta nada, tal vez una mejor ayuda económica.

Como puede observarse en los textos citados, el dinero es representado como un bien necesario para mejorar la calidad de vida propia y familiar; por tanto, el bienestar económico alude a la noción de mejoría en la calidad de vida y a la dimensión redistributiva de la justicia, con base en el mérito individual, como lo es finalizar una carrera universitaria y en consecuencia, obtener un buen empleo, se espera obtener una mayor riqueza .

Los textos anteriores reflejan la ideología de la sociedad del consumo, en la que la juventud se encuentra inmersa. Dicha cultura del consumo, se caracteriza por el uso, el disfrute, la complacencia y el gozo de los bienes y servicios poseídos, los cuales constituyen elementos centrales de un estilo de vida, en donde la capacidad de consumo determina el nivel de vida que posee la persona.

Asimismo, la pobreza es vista como el contraejemplo del ideal social de la vida adulta, constituyendo aquello que la juventud desea evitar, y reforzando a su vez, la conducta de estudiar. Esta idea fue encontrada en la respuesta de J. V. C. quien mencionó:

La misma situación que tenía ahí, por ejemplo, en ese sector de la escuela, a veces, o sea, cuando me iba en el autobús, o sea, es mucha la diferencia que hay en el centro [de la ciudad] y hacia ese sector, me refiero a la infraestructura y todo lo que se ve, y 
entonces el poder ver a diario, o sea, las casitas, las de cartón y niños jugando en la tierra, esten, a veces descalzos y con poca ropa, o sea, me ayudó, esten, como que a modificar mi pensamiento y, esten, y decir, o sea, tengo que decir algo, o sea, por mí, estén... ésta es la oportunidad que tengo, si no la aprovecho pues uno no quiera, pero pudiese terminar así y la misma situación que se vivía con mis compañeros, unos de... pues de bajos recursos y, a veces iban caminando a la escuela y, estén... me llegó y, estén... y al ver al ver eso y, esten, sí, sí decidí pues que quiero seguir estudiando y ser alguien, poder tener un buen empleo, o sea, tener la oportunidad de estudiar una licenciatura, ser un profesionista, terminar, y eso me hizo como que pensar en seguir estudiando.

Existe un pensamiento generalizado que dicta que si las oportunidades no son aprovechadas en la juventud, entonces no se llegará a ser alguien; en ese sentido, y como puede leerse en el texto anterior, estudiar el bachillerato adquiere el significado de "oportunidad", entendida esta como una circunstancia favorable, ya que a pesar de la situación de desventaja socioeconómica y cultural; se le ha posibilitado, en su etapa de juventud, al haber concluido su educación secundaria, en un momento oportuno en su vida; el ingreso a una escuela que pertenece a una universidad con prestigio social y calidad educativa, lo que le permitirá la obtención de un provecho: la competitividad, para lograr ciertos objetivos que consisten en ingresar a una Facultad de la misma universidad, terminar una carrera, mejorar su nivel de vida, entre otros.

Con base en Marx (1972), se encontró la existencia de una conciencia de clase entre el alumnado, al manifestar su pertenencia a grupos sociales en desventaja, considerando como lo justo, que la educación universitaria les permita dejar de ser personas desvalorizadas por su condición de pobreza. Este conocimiento de sí mismos como pertenecientes a una clase oprimida, en palabras de Freire (2002): "se encuentra perjudicado por su inmersión en la realidad opresora, ya que 'reconocerse' en antagonismo al opresor... no significa... luchar por la superación de la contradicción... uno de los polos... pretende, en vez de la liberación, la identificación con su contrario" (p. 37). Esto puede ser ejemplificado por lo mencionado por I. P. C.:

Las personas que entran a la preparatoria, algunas no tienen suficiente calidad económica, lo cual supongo que la universidad se puso en, cómo se puede decir, a 
pensar en nosotros, personas que tienen bajo nivel económico para que también puedan alcanzar sus sueños de engrandecer como personas.

En la reproducción de la ideología neoliberal dominante, los padres del alumnado del bachillerato juegan un papel importante, al comunicarle a sus descendientes sus deseos de progreso y su inconformidad con la pertenencia a una clase social baja, lo cual interiorizan sus hijos como un deseo propio. Como ejemplo de esto, se encuentra lo mencionado por M.T.A.: "Mis papás siempre me han dicho que sea yo, sea mejor que ellos, y ellos me impulsan a seguir y quiero estudiar, creo". Ella habla empleando las palabras de sus padres, y manifiesta duda personal en el deseo expresado por estudiar.

Acerca de la influencia paterna en la acción de estudiar, J. V. C. expresó que inicialmente estudiaba para evitar la represión de sus padres:

En ese entonces, hace tres años, lo hice no por satisfacción misma, sino por compromiso con mis papás; o sea, yo estudiaba sólo porque me decían, no, tienes que estudiar; en ese entonces pues yo tenía esa mentalidad, no, pues si no estudio mis papás me van a regañar.

C. M. R. también manifestó que sus padres ejercieron influencia en su decisión de estudiar el bachillerato: "Mis papás siempre han sido una pieza clave porque siempre me han dado motivos para seguir y ellos, apenas terminé la secundaria, me dijeron: no te puedes quedar sin estudiar, tienes que ingresar a la preparatoria".

Partiendo de teorías interaccionistas, los comentarios citados, muestran cómo la acción de estudiar el bachillerato es realizada para lograr la aceptación social, en este caso, la aceptación de los padres; es decir, la identidad subjetiva de este estudiantado, relacionada con ser estudiante universitario y tener una carrera profesional a nivel licenciatura, ha sido construida, en este caso, en una situación de interacción: la convivencia familiar, estando en negociación permanente con los otros: sus padres, de tal manera que la reivindicación que el alumnado hace de su self debe estar corroborada por sus padres, y viceversa, en palabras de Goffman (1997): "El self, por tanto, aparece sometido al escrutinio del acuerdo con el otro... el self es un 'efecto dramático', es decir, un producto de la escena representada, y no una causa de ella" (p. 269). El self se desvincula del estudio de su poseedor: "porque él y su cuerpo proporcionan simplemente la percha sobre la cual colgará durante cierto tiempo algo fabricado en colaboración" (Goffman, 1997, p. 269). 
Las enseñanzas de los padres han sido reforzadas por las autoridades del centro escolar, específicamente por la primera coordinadora general, mediante discursos que logran impregnar las mentes del alumnado con sentimientos de compasión o de culpa, refiriéndose al esfuerzo o sacrificio que realizan sus padres para que ellos/as puedan estudiar, de tal manera que los jóvenes son persuadidos a esforzarse en correspondencia al sacrificio de los padres. Al respecto, J. V. C. mencionó:

Recuerdo que hubo una plática ... donde, o sea, nos reunió a todos los estudiantes que éramos como doscientos, me imagino, que éramos de la primera generación y nos reunió y empezó a platicar con nosotros sobre el esfuerzo que nuestros papás hacen para la escuela y que nosotros estudiemos y, o sea, fue una plática muy, muy emotiva y entonces, o sea, tuve la fortuna de estar ese día ahí, y estén, y eso me ayudó mucho, estén, a recapacitar y ya pues, y a partir de ese día, o sea, pues dije: qué estoy haciendo, o sea, estoy desperdiciando la oportunidad de pues poder ser alguien y ya, a partir de ese día, estén, me puse las pilas.

Reforzando lo anterior, se encuentra lo afirmado por L. R. M.: "La maestra decía: cuando uno es estudiante no solamente tienes sobre tus hombros tus sueños, tienes los sueños de tu familia, de tus papás de verte convertido en una persona profesionista".

Desde esta perspectiva, los padres ejercen en sus hijos, al igual que el profesorado y las autoridades del centro escolar, lo que Bourdieu y Passeron (1998) llaman una acción pedagógica violenta; estos autores manifiestan que toda acción pedagógica: "es objetivamente una violencia simbólica en tanto que imposición, por un poder arbitrario, de una arbitrariedad cultural" (p. 45); esta acción pedagógica puede ser ejercida por todos los miembros educados de una formación social o grupo, por los miembros del grupo familiar a los que la cultura de un grupo o de una clase confiere esa tarea, o por el sistema de agentes explícitamente designados para ello por la educación institucionalizada; de esa forma, diversos agentes sociales contribuyen a la reproducción de la arbitrariedad cultural de las clases dominantes o de las dominadas.

El poder simbólico de la acción pedagógica no se reduce a la imposición de la fuerza, ya que sólo puede producir su efecto propiamente simbólico: "en cuanto que se ejerce en una relación de comunicación... y sólo puede producir su efecto propiamente pedagógico cuando... se dan las condiciones sociales de la imposición y de la inculcación" (Bourdieu y Passeron, 1998, pp. 45-47). La necesidad cultural, como necesidad cultivada, el asistir a la 
escuela, es producto de la autoridad pedagógica de la familia y/o de la escuela. Socialmente es visto positivamente por la familia, por la comunidad y por la sociedad en general, el pertenecer a una institución educativa que posee reconocimiento, ya que la buena imagen de esa institución, el pertenecer a ella, se le impregna a la persona, y dentro de una sociedad capitalista, el ostentar un certificado o título universitario expedido por una escuela con prestigio es necesario para tener valor dentro de la misma. Sin embargo, diversas realidades muestran, según Bechelloni (como se citó en Bourdieu y Passeron, 1998, p. 20):

Un segundo elemento, a lo mejor más importante, de contradicción, surge entre las expectativas que el sistema educativo (y en general cultural) suscita en términos de 'liberación', de autorrealización, de movilidad social vertical y la imposibilidad del sistema social (mercado del trabajo) y del mismo sistema escolar (mecanismos de selección y exclusión) de satisfacerlos en términos adecuados.

El permitir a los jóvenes el acceso a una institución educativa, no asegura la justicia social, pues como señalan Veleda, Rivas y Mezzadra (2011), la justicia educativa depende en primera instancia de lo que sucede fuera de la escuela, sobre todo de la estructura social establecida por los grupos hegemónicos, así como de las condiciones de vida de sus familias, de la infraestructura de servicios con los que cuentan en sus hogares y la atención de su salud. Lograr la justicia social implicaría transformaciones en los mecanismos meritocráticos bajo los cuales opera el sistema universitario, así como transformaciones en las relaciones que las escuelas mantienen con otras instituciones productivas para impactar su economía y calidad de vida personal.

En la figura 2 puede observarse un diagrama que integra los diversos significados y sentidos del bachillerato, desde la perspectiva del alumnado participante. 
Figura 2. Significados y sentidos del bachillerato en la vida presente y futura del alumnado de una escuela de nivel medio superior del sur de Mérida, Yucatán, México, diciembre de 2014.

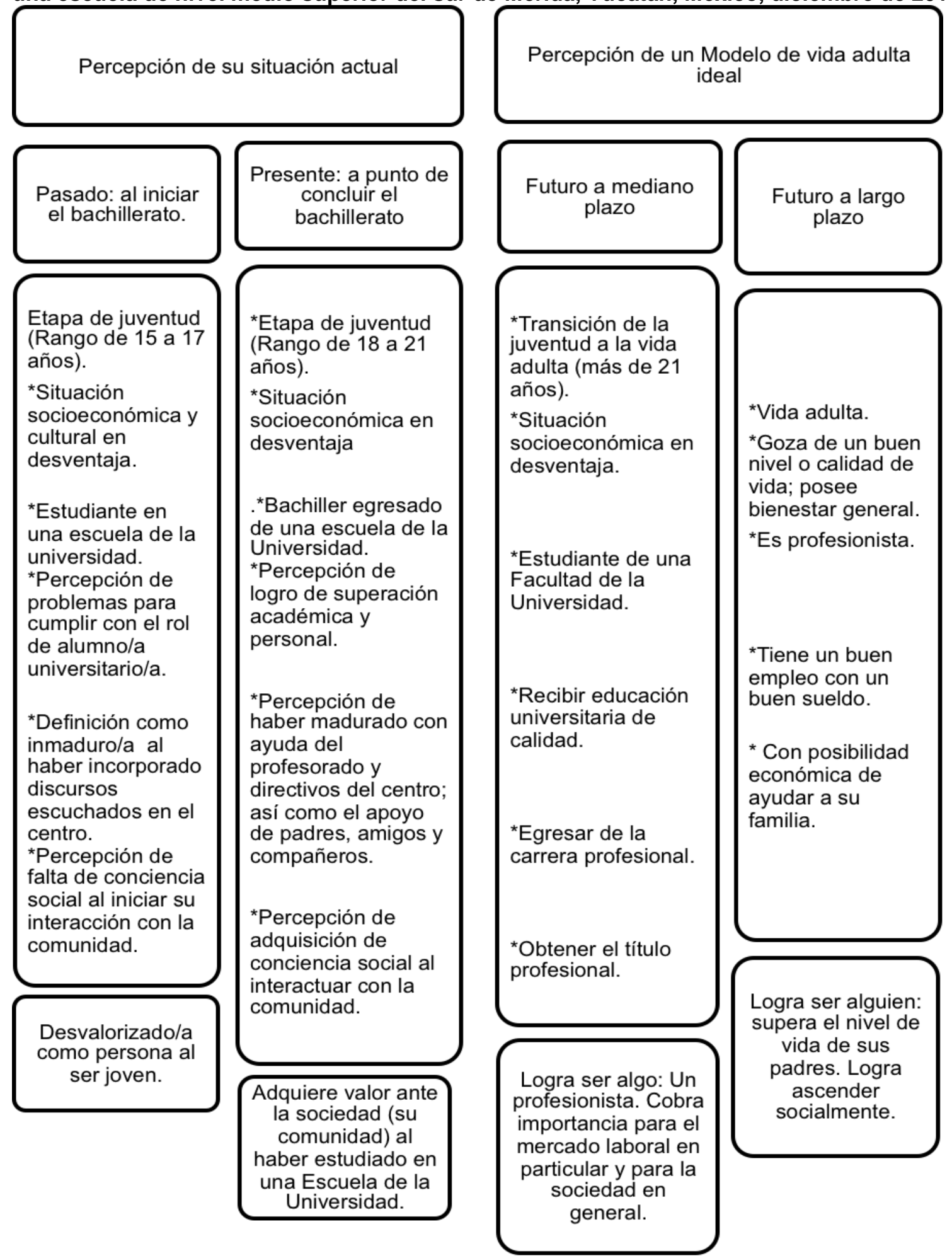

Fuente: Elaboración propia con la información resultante de la triangulación de los datos proporcionados por el alumnado participante. 


\section{Conclusiones}

Con base en los discursos del alumnado participante en la investigación, acerca de los significados y sentidos que tiene estudiar un bachillerato, fueron identificadas ideologías representativas de nociones de justicia social y económica asociadas a un modelo hegemónico de vida adulta, el cual está sustentado en el disfrute de una buena vida, donde el bienestar económico, el poder adquisitivo, un nivel de educación superior y un buen empleo, se consideran sus componentes. En este sentido, la buena educación representaría una noción de justicia educativa basada en la igualdad de oportunidades para el desarrollo de un capital cultural y humano que hace competitivo a cada estudiante en el mercado de trabajo, permitiendo el cambio de posición social como parte de la justicia socioeducativa; sin embargo, la complejidad de la estructura socio-económica neoliberal y capitalista, reflejada en el funcionamiento de los sistemas educativos, contribuye a la estratificación social, la marcación de desigualdades sociales y la naturalización de prácticas educativas y humanas injustas.

Como pudo observarse en las opiniones del alumnado que fungió como informante clave, actualmente en el imaginario social, el bachillerato significa una oportunidad para la juventud en desventaja socioeconómica y cultural; asimismo, el bachillerato adquiere un significado de instrumento para lograr en un tiempo futuro, una superación personal signada por el progreso económico e intelectual. Por parte de la dimensión distributiva de la justicia social, el aspecto económico es percibido por el alumnado como un factor necesario para lograr el reconocimiento en la sociedad, siendo la educación un medio válido y legítimo para lograrlo.

En conclusión, para el alumnado entrevistado tanto grupal como individualmente, el bachillerato se convierte en un instrumento de lucha social dada su condición de desventaja socioeconómica y cultural, cuya finalidad es el cumplimiento o el logro de un ideal de vida adulta, que ha sido incorporado desde la sociedad a su mundo ideológico; a partir de este punto de análisis es que puede identificarse un individualismo enmascarado de liberación solidaria.

En relación a la etapa de la juventud, como mencionan Tinoco y Evangelista (2006), la perspectiva de esta etapa como una transición y preparación para un futuro en el cual se tendrán oportunidades, tuvo sentido mientras esta etapa era más acotada en el tiempo; sin embargo, actualmente, con la prolongación de dicho período resulta difícil definir la finalización de esta etapa, la cual se ha ampliado hasta los 20, 25 ó 30 años. La 
desestructuración de la fase juvenil ha sido resultado de los cambios sociales y económicos, exigiendo mayores acreditaciones en un marco de menos opciones laborales, y al adelantarse la fase puberal y sociocultural de la adolescencia.

La invisibilidad ante los efectos negativos del modelo de estado neoliberal sobre sus propias vidas es un arma para que los grupos hegemónicos continúen reproduciendo la injusta distribución de bienes y servicios entre los miembros de la sociedad. Es necesario despertar la conciencia para liberarse de la opresión de ideologías que hacen a las personas cómplices de prácticas sociales y educativas injustas, siendo esto de suma relevancia sobretodo en un bachillerato de tipo comunitario, ya que en lo expresado por el alumnado participante, no estuvo presente la solidaridad y el compromiso con la comunidad.

Queda abierto a la discusión, al análisis y a la crítica, el discurso que le vende la educación a la juventud, como un instrumento liberador, basado en la distribución y el desarrollo de capacidades individuales sin cuestionar la falta de reconocimiento a la dignidad humana, independientemente de la clase social de procedencia, sobre todo cuando se tiene una estructura social basada en el reparto inequitativo de este bien común, dada la diversidad de niveles o grados de excelencia en las escuelas y las brechas de calidad entre unas y otras dependiendo de su ubicación geográfica.

El alcance de la investigación expuesta en este artículo refleja de manera comprensiva, el curriculum oculto de un grupo de jóvenes, quienes han incorporado y expresado una ideología que reproduce la meritocracia del siglo XX, en un mundo posmoderno basado en el placer, el individualismo y una estructura de estado neoliberal que choca con el discurso de la educación como bien social para lograr la justicia equitativa e igualitaria en las generaciones jóvenes y en otros grupos sociales diversos.

Queda mucho por trabajar en la línea de la justicia educativa, vinculando su estudio y consecución a través de prácticas políticas democráticas que acerquen cada día más las comunidades académicas y estudiantiles a las comunidades locales, ricas éstas en experiencias de vida y conocimientos tácitos que proporcionan situaciones auténticas, de las cuales se tiene mucho que aprender y compartir. Lograrlo no es fácil, es y será un camino inacabado, una constante búsqueda y transformación del sí mismo con las otras personas. Para ello, la inclusión, el diálogo y el aprendizaje cooperativo basado en el reconocimiento, el respeto, la tolerancia y el compromiso, con el rescate de la dignidad humana, más allá de la riqueza económica y el bienestar individual, es fundamental. 


\section{Referencias}

ATLAS.ti. (Versión 8). [Software de computación]. Berlín: Scientific Software Development $\mathrm{GmbH}$.

Bernstein, Basil. (1998). Pedagogía, control simbólico e identidad. España: Morata.

Bolívar Botia, Antonio. (2012). Justicia social y equidad escolar. Una revisión actual. Revista Internacional de Educación para la Justicia Social, 1(1), 9-45.

Bourdieu, Pierre. (1997). Capital cultural, escuela y espacio social. México: Siglo XXI Editores.

Bourdieu, Pierre y Passeron, Jean Claude. (1998). La reproducción. Elementos para una teoría del sistema de enseñanza. (3aㅡ ed.). México: Distribuciones Fontamara, S. A.

Cochran Smith, Marilyn y Demers, Kelly. (2009). Teacher Education as a Bridge? Unpacking Curriculum Controversies. En Michael Connelly, Ming Fang He y JoAnn Phillion. The Sage Handbook of Curriculum and Instruction (pp. 274-275). USA: Sage Publications.

Conde Gutiérrez del Álamo, Fernando. (2010). Análisis sociológico del sistema de discursos. Cuadernos metodológicos 43. España: Centro de Investigaciones Sociológicas.

Connell, Robert. (2006). Escuelas y justicia social (3 $3^{\underline{a}}$ ed). España: Morata.

Cortés Rodas, Francisco. (2005). Reconocimiento y justicia. Entrevista con Axel Honneth. Estudios Políticos, 27, 9-26.

Echazarreta González, Carlos. (2009, 27 de febrero). Boletín Informativo. Recuperado de http://www.uady.mx/sitios/prensa/boletines/feb-09/27-feb-09.html

Fascioli, Ana. (2011). Justicia social en clave de capacidades y reconocimiento. Revista de Filosofía, 23(1), 53-77.

Freire, Paulo. (2002). Pedagogía del oprimido (54ํㅡㄹ ed.). México: Siglo XXI editores.

Gimeno Sacristán, José. (2002). Educar y convivir en la cultura global. (2 $2^{\underline{a}}$ ed.). España: Morata.

Goffman, Erving. (1997). La presentación de la persona en la vida cotidiana. Buenos Aires: Amorrortu.

Heredia Soberanis, Norma, y Peniche Cetzal, Rubí (2016). Significados y sentidos de la gestión escolar en torno a la justicia educativa. Opción, 32(7). Recuperado de https://www.redalyc.org/articulo.oa?id=310/31048480055

Hernández-Castilla, Reyes., Euán Ramírez, Reyna e Hidalgo Farran, Nina. (2013). Prácticas del liderazgo Escolar implicado en la promoción de la justicia social: Estudio de un caso en la educación secundaria. Profesorado. Revista de currículum y formación del profesorado, 17(2). Recuperado de http://www.ugr.es/ recfpro/rev172COL3.pdf 
Martínez Rodríguez, Juan. (2005). Educación para la ciudadanía. España, Madrid: Morata.

Marx, Karl. (1972). Manuscritos: Economía y filosofía. Madrid: Alianza.

Ornelas Delgado, Jaime. (2007). Educación y neoliberalismo en México (2ª ed.). México: Benemérita Universidad Autónoma de Puebla.

Popkewitz, Thomas. (2000). Sociología política de las reformas educativas (3a ed.). España: Morata/ Fundación Paideia.

Rawls, John. (1995). Teoría de la justicia (2ª ed.). México: Fondo de cultura económica.

Secretaría de Educación Pública. (2007). Programa sectorial de educación 2007 - 2012. México: Talleres de la Comisión Nacional de Libros de Texto Gratuitos: Autor.

Sen, Amartya. (1995). Nuevo examen de la desigualdad. Madrid: Alianza Editorial.

Sen, Amartya. (2000). Desarrollo y libertad. Barcelona: Planeta.

Simons, Helen. (2011). El estudio de caso: Teoría y práctica. España: Morata.

Stake, Robert. (2007). Investigación con estudio de casos. (4ª ed.). España: Morata.

Tinoco, Rolando y Evangelista, Angélica. (2006). La problemática de inserción de las y los jóvenes del Sur - Sureste. En José Pérez Islas y Mónica Valdés González. (Coords.). Jóvenes Mexicanos. Encuesta Nacional de Juventud 2005 (pp. 175-207). México: Instituto Mexicano de la Juventud.

Torres Santomé, Jurjo. (2009). La justicia curricular. El caballo de Troya de la cultura escolar. España: Morata.

Universidad Autónoma de Yucatán (UADY). (2010). Plan de Desarrollo Institucional 2010 2020. Yucatán, México: Autor.

Veleda, Cecilia. Rivas, Axel y Mezzadra, Florencia. (2011). La construcción de la justicia educativa. Criterios de redistribución y reconocimiento para la educación argentina. Buenos aires, Argentina: CIPPEC/ UNICEF/ Embajada de Finlandia. 
Revista indizada en

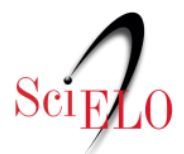

redalyc sy latindex

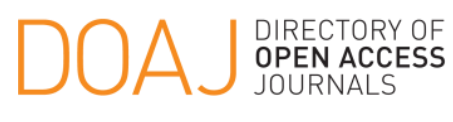

Distribuida en las bases de datos:

- Dialnet

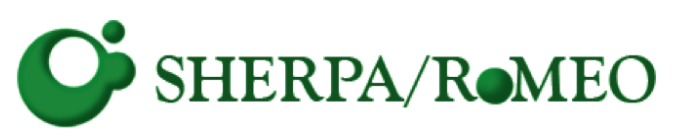

REDIB

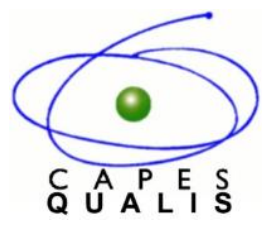

MIAR 